\title{
Estudio de la protección del refuerzo de partículas de SiC mediante barreras activas por sol-gel en materiales compuestos de matriz de
}

\author{
A. UREÑA, J. RAMS, M. D. ESCALERA Y M. CAMPO \\ Dpto. de Ciencias Experimentales e Ingeniería, Escuela Superior de Ciencias Experimentales e Ingeniería \\ Universidad Rey Juan Carlos, C/ Tulipán s/n, Móstoles 28933, Madrid
}

\begin{abstract}
Con objeto de prevenir la degradación y mejorar la mojabilidad de los materiales compuestos de matriz de aluminio reforzados con partículas de $\mathrm{SiC}$ se ha desarrollado un recubrimiento microcristalino, homogéneo y transparente de $\mathrm{SiO}_{2}$ obtenido por el método sol-gel. La eficacia del recubrimiento como barrera se ha comprobado realizando ensayos de fusión a diferentes tiempos y temperaturas sobre pastillas de material compuesto de matriz de aluminio obtenidas por compactación en frío de polvos. El recubrimiento limita la formación de $\mathrm{Al}_{4} \mathrm{C}_{3^{\prime}}$ lo que indica que la resistencia a la degradación del nuevo material es muy superior a la del fabricado con partículas de SiC sin recubrir.

\section{Palabras clave: $\mathrm{SiC}$, aluminio, materiales compuestos, silice, sol-gel, reactividad}

\section{Protection study of aluminium matrix composites reinforced with SiC particulates coated with sol-gel active barriers}

A microcrystalline, homogeneous and transparent sol - gel silica coating has been developed to avoid the degradation and improve the wettability of aluminium matrix composites reinforced with $\mathrm{SiC}$ particles. Composite aluminium matrix samples have been made by cold powder compaction, and the coating efficiency has been checked by melting the samples several temperature and times. The coating reduces the appearance of $\mathrm{Al}_{4} \mathrm{C}_{3}$, showing that the degradation resistance of the new material is much higher than that of the uncovered $\mathrm{SiC}$ particles composite one.
\end{abstract}

Keywords: SiC, aluminium, composites, silica, sol-gel, reactivity

\section{INTRODUCCIÓN}

Los materiales compuestos de matriz de aluminio reforzados con partículas de $\mathrm{SiC}$ mantienen la ligereza propia de las aleaciones de aluminio y presentan mejores propiedades mecánicas. A pesar de ello, la reducida mojabilidad existente entre el $\mathrm{SiC}$ y el aluminio fundido a temperaturas bajas ha limitado el uso de los métodos de fabricación por moldeo, que son los más utilizados industrialmente por su facilidad de procesado y bajo coste. Este problema se podría subsanar aumentando la temperatura de procesado, pero la reacción que tiene lugar entre la matriz y el refuerzo origina la formación de $\mathrm{Al}_{3} \mathrm{C}_{4^{\prime}}$ que es un material frágil y sensible al contacto con la humedad, por lo que degrada el material compuesto. Para evitar su formación se emplean recubrimientos sobre el refuerzo que actúan de barrera protectora (1). Diferentes recubrimientos (metálicos y cerámicos), así como múltiples métodos de síntesis (oxidación, electroless, sol - gel) $(2,3)$ se han utilizado. Uno de ellos es sílice obtenida por oxidación directa del SiC que, además de ser un buen recubrimiento protector, aumenta la mojabilidad de las partículas por el aluminio fundido $(4,5,6)$. Su principal problema se encuentra en que por su delgadez se agota en periodos de tiempo no muy elevados. La técnica sol - gel permite obtener recubrimientos cerámicos de múltiples materiales partiendo de un gel precursor preparado por hidrólisis en condiciones ácidas de una disolución alcohólica del alcóxido de diferentes metales (7). Con esta técnica se ha depositado un recubrimiento homogéneo y uniforme de $\mathrm{SiO}_{2}$ sobre partículas de $\mathrm{SiC}$ (8). En el trabajo se caracteriza este recubrimiento y se evalúa su eficacia como barrera protectora en materiales compuestos de aleación de aluminio mediante la realización de ensayos de fusión que simulan los procesos de colada.

\section{PARTE EXPERIMENTAL}

\subsection{Recubrimiento de las partículas por sol-gel}

Los soles híbridos de $\mathrm{SiO}_{2}$ se prepararon a partir del precursor tetraetiletoxisilano (TEOS) disuelto en etanol y agua destilada. La hidró- lisis se ha llevado a cabo durante 2 horas a temperatura ambiente, $\mathrm{pH}$ ácido y la concentración del sol fue de $204 \mathrm{~g} / \mathrm{l}$. Una vez recubiertas las partículas de $\mathrm{SiC}$ se secaron a $120^{\circ} \mathrm{C}$ y se sometieron a una tratamiento térmico final. Se ha estudiado la influencia en las propiedades y características del recubrimiento de $\mathrm{SiO}_{2}$ de diferentes parámetros del proceso sol-gel. Se han usado dos catalizadores ácidos $\left(\mathrm{HCl}, \mathrm{HNO}_{3}\right)$, varias relaciones molares $\mathrm{H}_{2} \mathrm{O} /$ TEOS $(1,2,3,4$ y 5$)$ y diferentes temperaturas de tratamiento térmico $\left(500-900{ }^{\circ} \mathrm{C}\right)$. La caracterización de los diferentes recubrimientos obtenidos ha dado como resultado que el recubrimiento más compacto y uniforme se obtiene con una relación 5 molar $\mathrm{H}_{2} \mathrm{O}$ / TEOS, catalizado con una disolución $\mathrm{HCl}$ 0,1 M, y tras un tratamiento térmico a $500^{\circ} \mathrm{C}$.

\subsection{Ensayos de fusión}

Los ensayos de fusión realizados tuvieron como base el estudio del efecto protector que tiene el recubrimiento de $\mathrm{SiO}_{2}$ obtenido por sol - gel. Se prepararon pastillas compuestas por compactación en frío de polvos de aleación de aluminio AA6061 (de Aluminium Powder Company) con un $20 \%$ de partículas de SiC (F360 de Navarro SiC) en estado de recepción y protegidas con el recubrimiento de sílice obtenido por sol - gel.

Los ensayos se realizaron en un horno de vacío $\left(2,5 \cdot 10^{-4} \mathrm{~Pa}\right)$ dentro de un crisol de alúmina. El ciclo de fusión constaba de una etapa de calentamiento de 30 minutos hasta alcanzar la temperatura elegida a la que se mantenía diferentes intervalos de tiempo. El mismo ensayo se repetía con partículas en estado de recepción y recubiertas. Se han hecho ensayos a $800{ }^{\circ} \mathrm{C}$ de 20 y 40 minutos, a $850{ }^{\circ} \mathrm{C} 30$ minutos y a 900 ${ }^{\circ} \mathrm{C} 60 \mathrm{~min}$. Para evaluar el grado de reacción del aluminio fundido con las partículas de $\mathrm{SiC}$ se disolvió electrolíticamente la matriz de aluminio tras los ensayos de fusión, empleando para ello una disolución de $\mathrm{HNO}_{3}$ (30\% en volumen) en metanol y voltajes de 10 a $15 \mathrm{~V}$. 


\section{RESULTADOS Y DISCUSIÓN}

\subsection{Caracterización del recubrimiento}

La figura 1 muestra la imagen de SEM de una partícula recubierta por el método del sol - gel, su superficie lisa y aristas redondeadas sugieren la presencia de un recubrimiento homogéneo y uniforme. El espectro de microanálisis EDX realizado sobre las partículas en estado de recepción (fig. 2a) muestra sólo los picos del Si y del C, mientras que en las partículas tratadas (fig. $2 \mathrm{~b}$ ) se observa la presencia de oxígeno de la sílice del recubrimiento. La microestructura del recubrimiento se ha estudiado mediante difracción de rayos X (fig. 3), observándose un pico muy ancho centrado en $22^{\circ}$ que es típico del vidrio de sílice microcristalino que se obtiene por el método sol-gel. Por último, la figura 4 muestra una micrografía TEM en la que se aprecia una partícula de $\mathrm{SiC}$ con una pequeña parte del recubrimiento (en este caso de $150 \mathrm{~nm}$ de espesor de un total estimado de $0,7 \mu \mathrm{m}$ ) que se mantiene adherida a la partícula tras el proceso de molienda de preparación de la muestra para microscopía. La difracción de electrones realizada sobre el recubrimiento (recuadro inferior) confirma que se trata de sílice microcristalina. Esto mismo se aprecia mediante la espectroscopia fotoelectrónica de rayos X (XPS). El orbital $2 p$ del Si en el SiC en estado de recepción (fig. 5) muestra la línea de emisión del enlace del

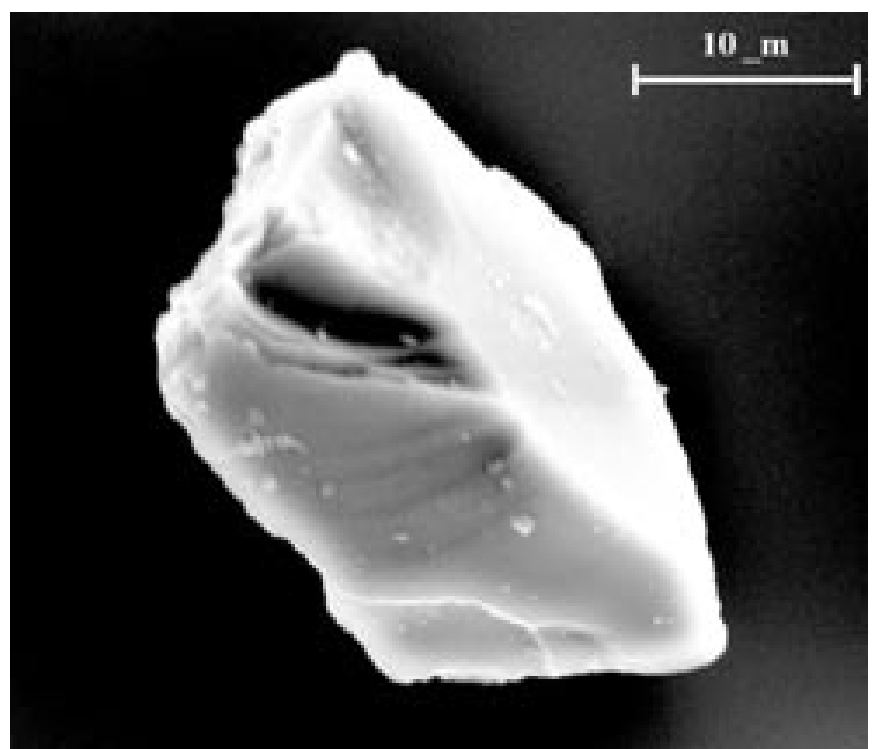

Fig. 1: Imagen SEM de una partícula recubierta con sílice obtenida por sol-gel.

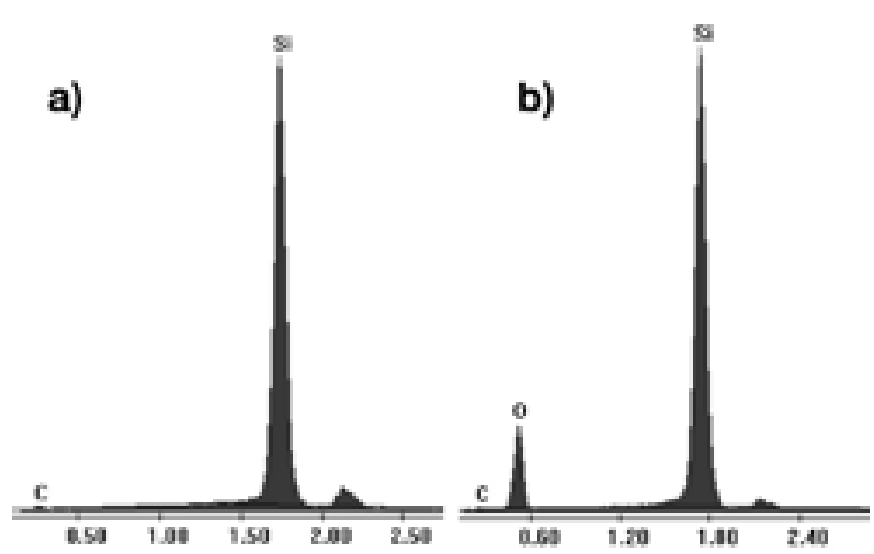

Fig. 2: Espectro de microanálisis EDX de la partícula de $\mathrm{SiC}$ a) en estado de recepción b) con recubrimiento de $\mathrm{SiO}_{2}$ sol-gel.
Si-C a 100,7 eV y las líneas de emisión de los enlaces Si-O-C y Si-O de productos intermedios (oxicarburos $\mathrm{SiO}_{\mathrm{x}} \mathrm{C}_{\mathrm{y}}$ ) debidos a la oxidación de la superficie de la partícula con la atmósfera a energías superiores. En las partículas recubiertas por sol-gel se observan las líneas de emisión del enlace Si-C a 100,7 eV y las del Si-O 103,4 eV de la capa de sílice. Comportamientos similares, aunque menos acusados, se observan en el orbital 1s del C.

Para la caracterización del proceso de sinterización del recubrimiento se han realizado análisis térmicos diferenciales (ATD) a las partículas en estado de recepción y recubiertas; restando ambas se ha obtenido el análisis del recubrimiento (fig. 6). Desde la temperatura ambiente hasta $\operatorname{los} 150^{\circ} \mathrm{C}$ se puede apreciar un pico endotérmico originado por la evaporación del agua y del alcohol atrapado en la estructura y en los poros de la sílice sol - gel. Los dos pequeños picos endotérmicos a $175{ }^{\circ} \mathrm{C}$ y $225^{\circ} \mathrm{C}$ se deben a la descomposición de residuos orgánicos que se forman durante el proceso de gelificación y se solapan con la ancha curva exotérmica presente entre $170{ }^{\circ} \mathrm{C}$ y $450{ }^{\circ} \mathrm{C}$ debida a la sinterización de los microporos del material. Esta interpretación está avalada por las medidas de superficie específica (BET) que muestran que los tratamientos térmicos realizados a $500{ }^{\circ} \mathrm{C}$ y, en mayor medida a $800^{\circ} \mathrm{C}$, producen un material compacto con una superficie específica que puede hacerse inferior a la del $\mathrm{SiC}$ inicial.

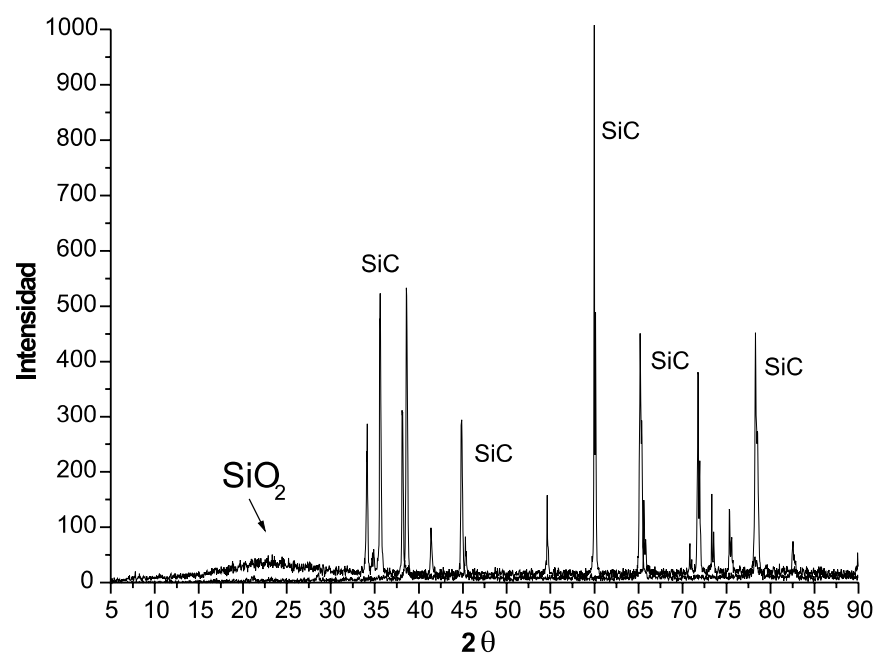

Fig. 3: Difractograma de rayos $\mathrm{X}$ de las partículas recubiertas.

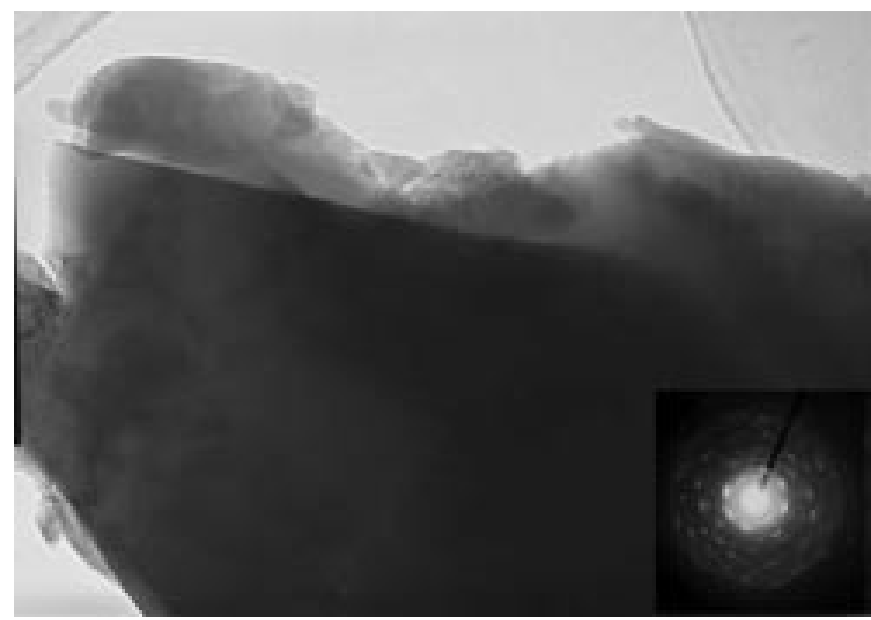

Fig. 4: Imagen TEM de una partícula recubierta y difracción de electrones sobre la sílice. 

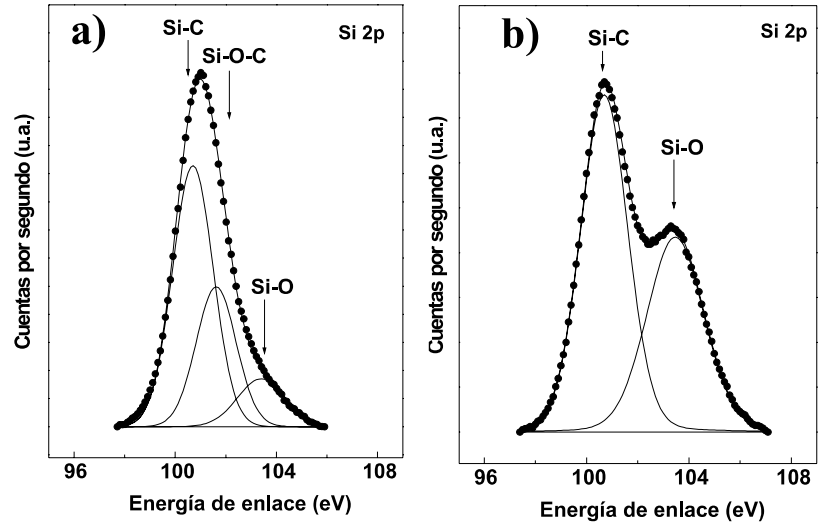

Fig. 5: Espectro XPS del orbital 2p del Si. a) partículas en estado de recepción; b) partículas recubiertas con $\mathrm{SiO}_{2}$ sol-gel.

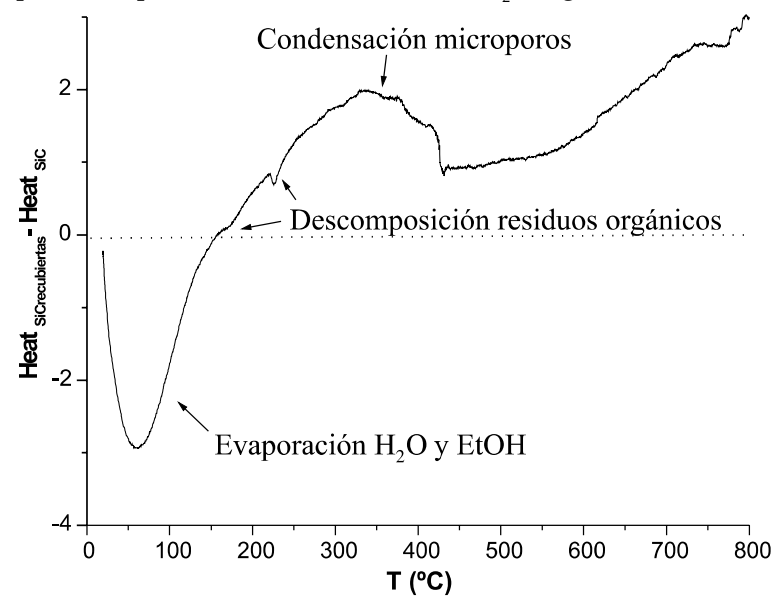

Fig. 6: Transferencias de calor del recubrimiento de sílice sol-gel.
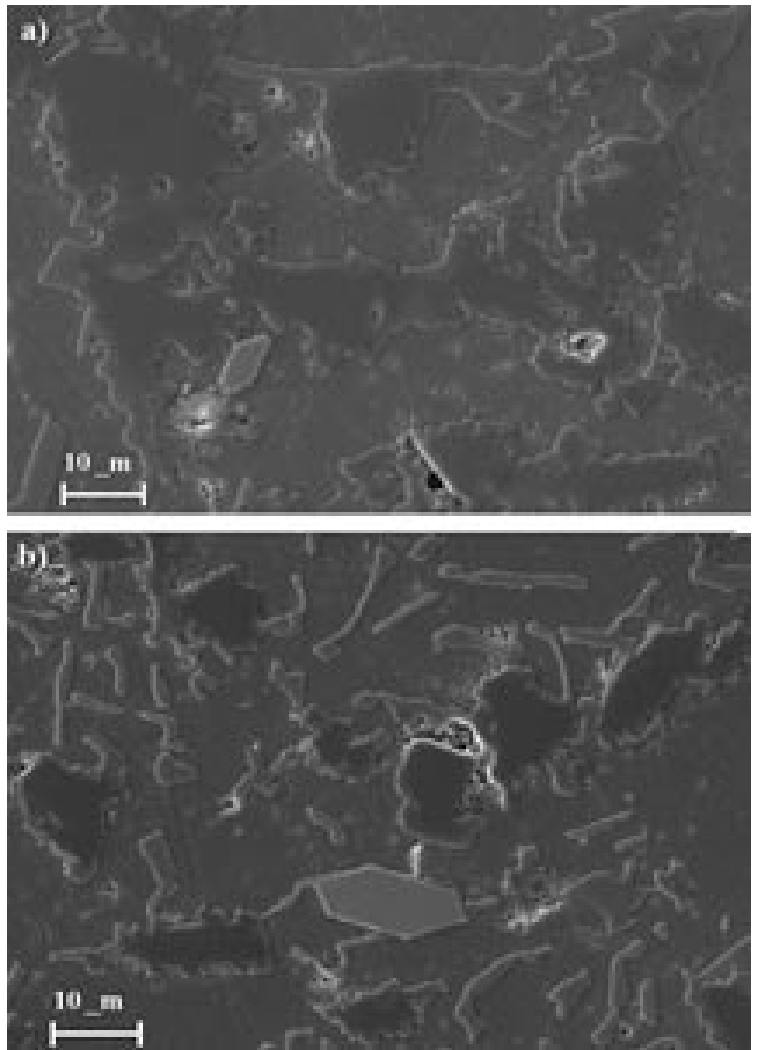

Fig. 7: Imágenes de SEM del material compuesto sometido a un ensayo de $850^{\circ} \mathrm{C}$ y $30 \mathrm{~min}$ fabricado con a) partículas sin proteger y b) partículas con el recubrimiento sol-gel.

\subsection{Estudio de la reactividad}

Los ensayos de reactividad se realizaron siguiendo el método descrito en el apartado 2.2 y las muestras ensayadas se sometieron a diferentes estudios. La figura 7a muestra una imagen de SEM del material fabricado con las partículas de $\mathrm{SiC}$ iniciales y la figura $\mathrm{7b}$ corresponde al material obtenido con partículas recubiertas. Las partículas sin recubrir han sido atacadas por el aluminio, han perdido su forma original y se encuentran rodeadas por productos de reacción. Por el contrario, las partículas recubiertas han resistido en gran medida el proceso y sólo es posible ver la reacción en zonas localizadas, fenómeno que puede deberse a la ruptura local de la barrera. Además, como se puede apreciar en las imágenes, la mojabilidad de las partículas recubiertas es muy superior a la de las partículas en estado de recepción. Una evaluación del grado de ataque se ha realizado utilizando difracción de rayos $\mathrm{X}$ como se muestra en la figura $8 \mathrm{a}$, correspondiente al material de referencia, y en la figura $8 \mathrm{~b}$ al material fabricado con partículas recubiertas. En todas las condiciones de tiempo y temperatura los picos del $\mathrm{Si}$ y del $\mathrm{Al}_{4} \mathrm{C}_{3}$ en el material inicial son muy superiores a los que aparecen en el caso de las partículas recubiertas, siendo los picos apenas perceptibles incluso en los tratamientos a $850^{\circ} \mathrm{C}$. Su aparición
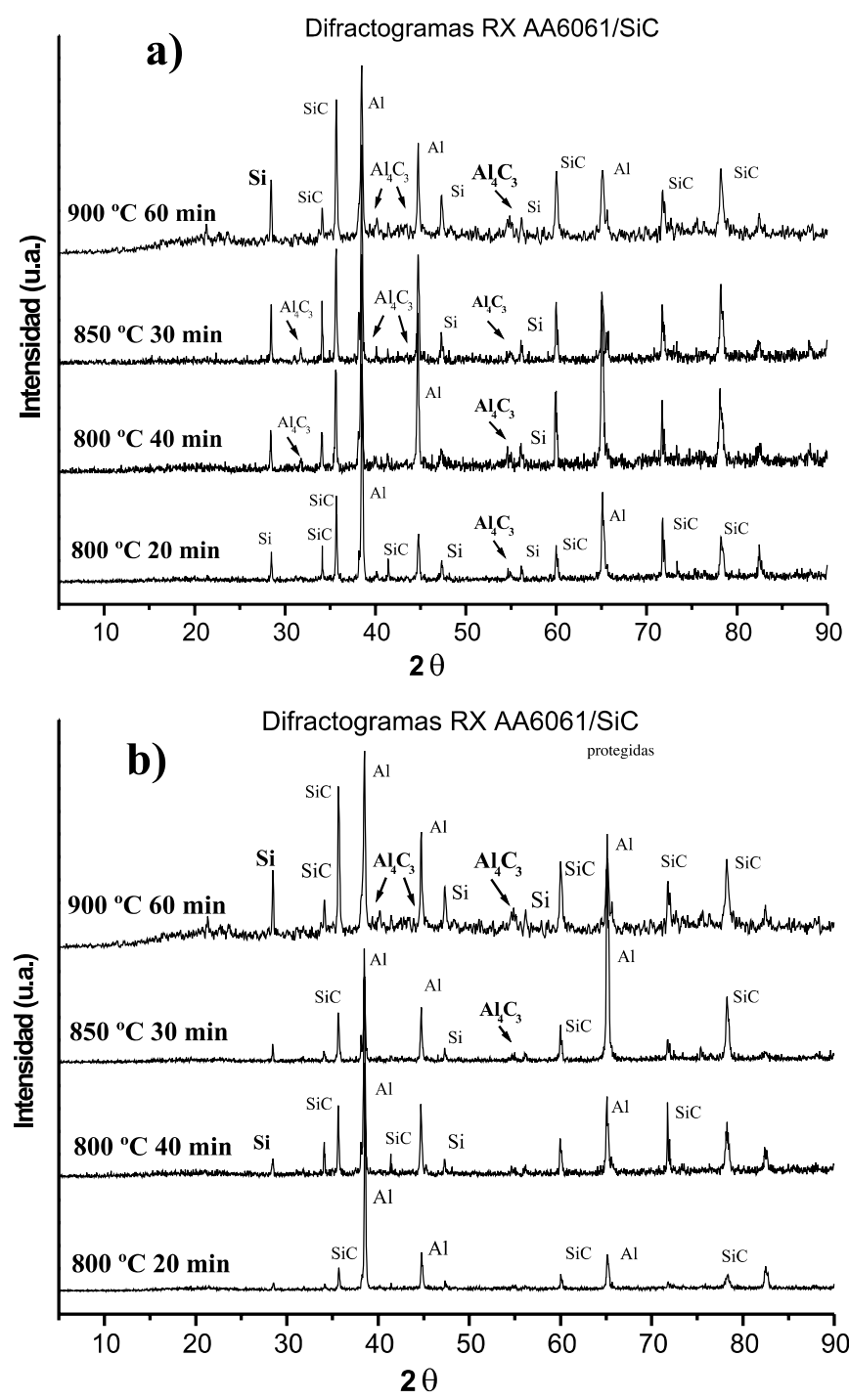

Fig. 8: Difractogramas de RX de los ensayos de fusión en las diferentes condiciones de temperatura y tiempo de las pastillas de aleación A6061 con a) partículas sin proteger y b) partículas protegidas con la sílice sol-gel. 
a tiempos y temperaturas elevados indica que la barrera se agota, lo que implica que se trata de una barrera activa que reacciona con el aluminio produciendo aluminosilicatos, aunque cuando la barrera se ha enriquecido mucho en aluminio puede reaccionar con la partícula de $\mathrm{SiC}$ y se produce la formación de $\mathrm{Si}$ y de $\mathrm{Al}_{4} \mathrm{C}_{3}$.

Finalmente se estudió la morfología superficial del ataque del aluminio sobre las partículas mediante su extracción con técnicas electrolíticas de la matriz metálica. La figura 9a muestra el fuerte ataque que sufren las partículas sin recubrir, apreciándose la disolución preferente de algunos planos cristalinos característica de este proceso $(5,6)$. La figura $9 \mathrm{~b}$ confirma el análisis de los difractogramas de rayos $\mathrm{X}$ y las imágenes del material compuesto, ya que muestra que las partículas recubiertas por sol-gel sufren una degradación muy inferior y que sólo aparece tras el agotamiento de la barrera. Recubrimientos de mayor espesor permitirían aguantar el contacto con el aluminio fundido durante más tiempo.

\section{CONCLUSIONES}

Se han determinado las condiciones idóneas para recubrir las partículas de $\mathrm{SiC}$ con una capa compacta y homogénea de $\mathrm{SiO}_{2}$ microcristalina mediante la técnica del sol-gel. Se ha demostrado que este recubrimiento actúa como una barrera activa aumentando la mojabilidad de las partículas por el aluminio fundido y, simultáneamente, impidiendo la formación del $\mathrm{Al}_{4} \mathrm{C}_{3}$ que degrada las propiedades de los materiales compuestos de matriz de aluminio. Este recubrimiento resiste temperaturas de hasta $850{ }^{\circ} \mathrm{C}$ pero se agota en procesos prolongados, aunque recubrimientos de mayor espesor permitirían aumentar el tiempo que resistirían las partículas.

\section{AGRADECIMIENTOS}

Los autores agradecen a la CICYT (proyecto MAT2000-1646-C0201) y a la URJC por la financiación proporcionada.

\section{BIBLIOGRAFÍA}

1. T.P.D. Rajan, R.M. Pillai, B.C. Pai. "Review reinforcement coatings and interfaces in aluminium metal matrix composites". J. Mater. Sci. 33, 3491 - 3503 (1998).

2. M. Suéry, G. L'Espérance, B.D. Hong, L. Nguyen Thanh, F. Bordeaux. "Development of particulate treatments and coatings to reduce SiC degradation by liquid aluminium". J. Mat. Eng. \& Perf. 2 (3), 365-374 (1993).

3. B. Kindl, Y.H. Teng, Y.L. Liu. "Protective coatings for commercial particulates". Composites. 25 (7), 671-676 (1994).

4. Zhongliang Shi, J.M. Yang, J.C. Lee, Di Zhang, H.I. Lee, Renjie Wu. "The interfacial characterization of oxidized $\mathrm{SiC}_{\mathrm{p}} / 2014 \mathrm{Al}$ composites". Mat. Sci. and Eng. A303, 46-53 (2001).

5. A. Ureña, J.M. Gómez de Salazar, L. Gil, P. Rodrigo, E.E. Martínez, J.L. Baldonedo, E.Criado. "Estudio de la reactividad entre aleaciones de aluminio y partículas de $\mathrm{SiC}$ en estado de recepción y previamente oxidadas" Bol. Soc. Esp. Ceram. V., 39 (2) (2000).
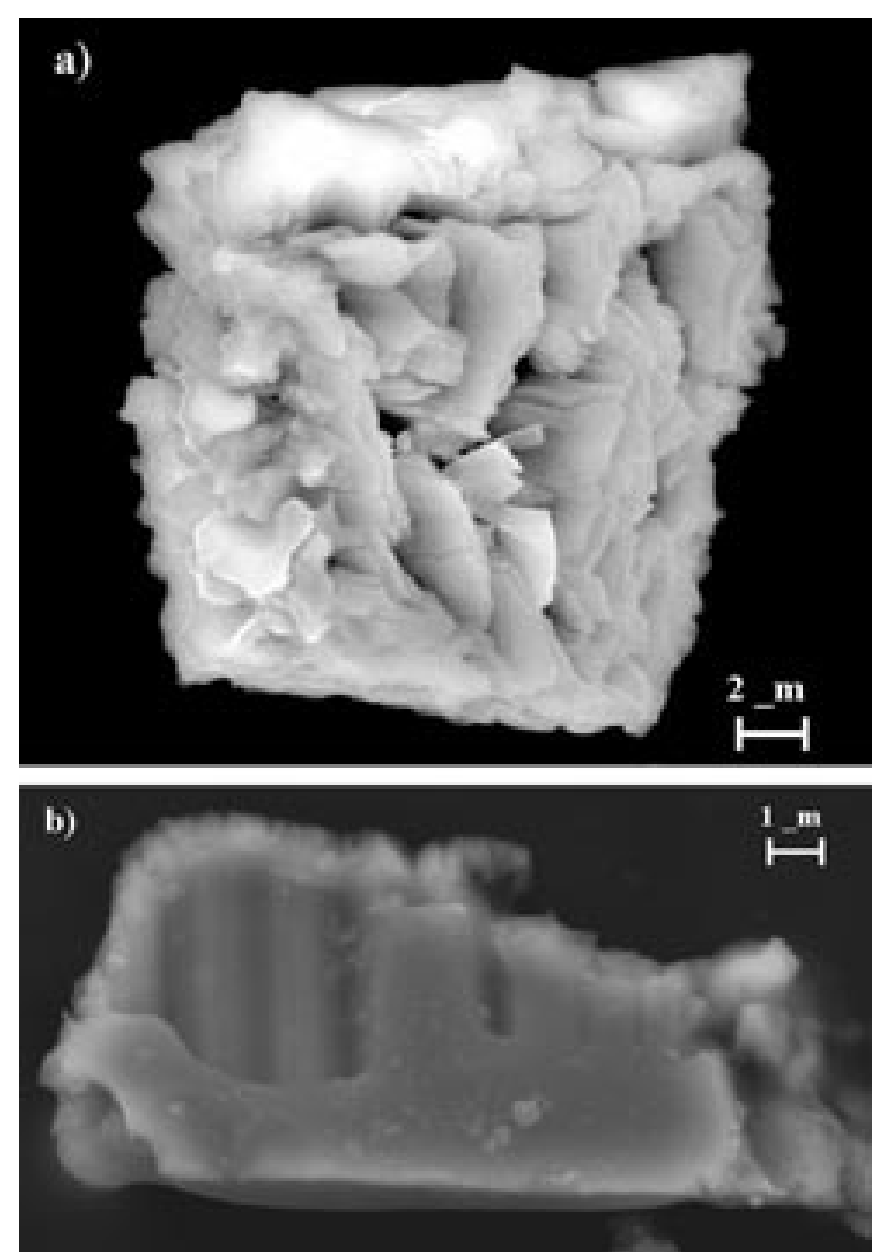

Fig. 9: Imágenes de SEM de la morfología superficial del ataque de las partículas de $\mathrm{SiC}$ después de ensayarlas a $850^{\circ} \mathrm{C}$ y $30 \mathrm{~min}$. a) partículas sin proteger; b) partículas protegidas con el recubrimiento de $\mathrm{SiO}_{2}$ sol-gel

6. A. Ureña, M.D. Escalera, P. Rodrigo, J.L. Baldonedo, L. Gil. “Active coating for $\mathrm{SiC}$ particles to reduce the degradation by liquid aluminium during processing of aluminium matrix composites: study of interfacial reactions". J. Microscopy. 201, 1-16 (2000).

7. C.J. Brinker, G.W.Scherer. "Sol-Gel sciencie: the physics and chemistry of sol-gel processing". A. Taylor, and J.A. Fernie. (1997), A Review of Sol-Gel Technology for the fabrication of glasses and ceramics.

8. M.Campo, J.Rams, L.Gil, A.Ureña. "Recubrimiento de los refuerzos de partículas de $\mathrm{SiC}$ con $\mathrm{SiO}_{2}$ mediante la técnica sol-gel para la fabricación de material compuesto de matriz de aluminio". MATCOMP’01, Gijón 2001.

Recibido: 1.2 .03

Aceptado: 30.11 .03 